\title{
A study on the construction of disaster information contents for the Storm and Flood Damage Insurance Map
}

\author{
J. Kang ${ }^{1}$, S. Yeon ${ }^{1 *}$, J. Lee $^{1}$ \\ ${ }^{1}$ Spatial Information Research Institute, Korea Land And Geospatial InformatiX Corporation, (kangdaejang, yeon, jaslee)@1x.or.kr
}

Commission VI, WG VI/4

KEY WORDS: Insurance Map, Public data, Linkage System, Disaster, Information Contents

\begin{abstract}
:
Typical types of natural disasters that occur in Korea are damages from heavy rain, storm, and heavy snow. In order to prepare for this, the storm and flood damage insurance program is operated. For this purpose, the risk of these damages is calculated for each region, and the storm and flood damage insurance map is created based on the risk. This map can provide insight into the degree of risk to wind and flood, snow damage, as well as policies to prevent and prepare for each type of natural disaster. In order to support decision-making by utilizing this insurance map, it is necessary to use with disaster Information contents. In order to efficiently construct such disaster information contents, it is possible to utilize public data produced by various organizations. Korea has a public data portal to open various administrative information. The public data portal currently publishes and updates about 25,000 data from 700 organizations. In this study, the linkage system is designed that can construct disaster information contents by collecting public data and processing it so that it can be overlapped with the insurance map. The system automatically links public data to keep up-to-date disaster information content. It is expected that it will be able to prevent and prepare for natural disaster by supporting the decision making of decision makers related to flood damage.
\end{abstract}

\section{INTRODUCTION}

The last 10 years, the damage incurred by the causes of natural disasters in Korea has been $52 \%$ for heavy rain, $26 \%$ for storm, and $20 \%$ for heavy snow, and $1 \%$ for strong wind and wind wave. (MPSS 2016). In order to respond to damage from heavy rain, storm, and heavy snow which account for most of these natural disasters, the Storm and Flood Damage Insurance (SFDI) program has been operating in Korea. SFDI is a policy insurance administered by the central government and operated by private insurers (Lee and Lee, 2017). National and local governments support some of the premiums so that citizens can use them at low rates. Through this, they will actively cope with the unexpected storm and flood damage.

For the reasonable operation of the flood damage insurance, individual rates are being developed which can be applied differently according to the risk due to storm and flood by region and the risk by types of facilities subject to insurance (Lee and Lee, 2015). In order to manage such information, SFDI map is being produced and the integrated management system is being developed (Hwang et al., 2015).

In order to increase the availability of the integrated management system for SFDI map and to support decision making for disaster management, various additional information is needed. The Storm and Flood Damage Information Content (SFDIC) is variety of information that can be used for disaster management together with SFDI map. This study suggested efficient SFDIC construction method using public data opened by the government. And designed a linkage system to collect and convert open public data into SFDIC.

\section{CONSTRUCTION METHOD OF SFDIC}

\subsection{Public Data Portal Status}

Public data refers to data on the whole of the national life, including economic, geographical, transportation, statistics, weather, culture, etc. (Yeon et al., 2015). Recently, the importance of public data has increased, and public data portals have been established in the United States, Australia, and other countries around the world to provide public data. In Korea, the public data portal for managing and opening public data was established in accordance with the Public Data Opening Act of 2013. Currently, about 24,824 data provided by 697 organizations are managed in the public data portal. And there are three ways to provide public data, such as File download, Open API, Standardization data. (Table 1).

\begin{tabular}{|l|l|c|}
\hline \multicolumn{2}{|l|}{ Item } & Quantity \\
\hline \multicolumn{2}{|l|}{ Data Provider (Public Institutions) } & 697 \\
\hline Type of & File Download Data & 22,255 \\
Providing & Open API & 2,511 \\
& Standardization Data & 58 \\
\hline
\end{tabular}

Table 1. Opening status of public data portal

\subsection{Utilization of Public Data}

Summarizing the available data on the public sector data portal as shown in Table 2. Among the 17 areas, SFDIC can be used for Disaster \& Safety, Healthcare, and Transport \& Logics. There are 5,850 data in the field, which is about a quarter of the total data. Of these data, the actual usable data may be small. However, because the areas and numbers of data openings are

\footnotetext{
* Corresponding author
} 
constantly increasing, and keeping up-to-date data it is valuable enough to be used as SFDIC.

\begin{tabular}{|l|c|c|}
\hline Sector & Quantity & Ratio \\
\hline & $\#$ & $\%$ \\
Education & 1,304 & 5.25 \\
Land Management & 1,034 & 4.17 \\
Public Administration & 3,823 & 15.40 \\
Finance & 467 & 1.88 \\
Employment & 1,860 & 7.49 \\
Social Welfare & 1,610 & 6.49 \\
Food \& Health & 439 & 1.77 \\
Culture \&Tourism & 4,289 & 17.28 \\
Healthcare \& Medical & 1,666 & 6.71 \\
Disaster \& Safety & 1,918 & 7.73 \\
Transport \& Logistic & 2,221 & 8.95 \\
Environment \& Weather & 1,905 & 7.67 \\
Science \& Technology & 912 & 3.67 \\
Agriculture \& Fishery \& & 1,044 & 4.21 \\
Livestock & 251 & 1.01 \\
National Security & 65 & 0.26 \\
Law & 16 & 0.06 \\
Etc & 24,824 & 100 \\
Total
\end{tabular}

Table 2. Quantities and ratios of data by sector

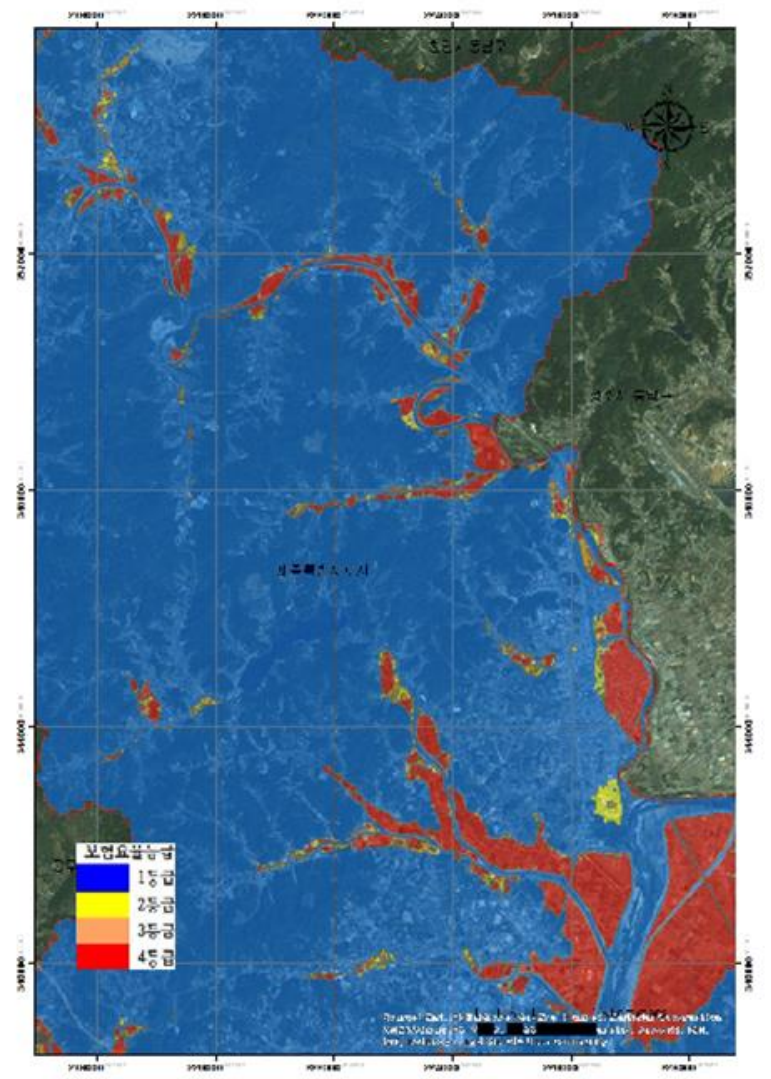

Figure 1. Example of the SFDI Map (Sejong in Korea)

\subsection{SFDI Map and SFDIC}

The SFDI map is constructed based on the spatial information data of the polygon type with the attribute of the risk in step 4 as shown in Figure 1. Therefore, in order to utilize open public data to overlap with SFDI map, location-matching information is essential. In general, locations are matched using information such as coordinates, address, and zip code. As a result of reviewing the public data, there are few information including coordinates which require expert knowledge, but a lot of data referring to address or postal code has been confirmed. Such data can be converted into spatial information data using coordinate information matched with address or zip code, and can be used as SFDIC.

\section{LINKAGE SYSTEM FOR SPDIC CONSTRUCTION}

\subsection{Establishment of linkage process}

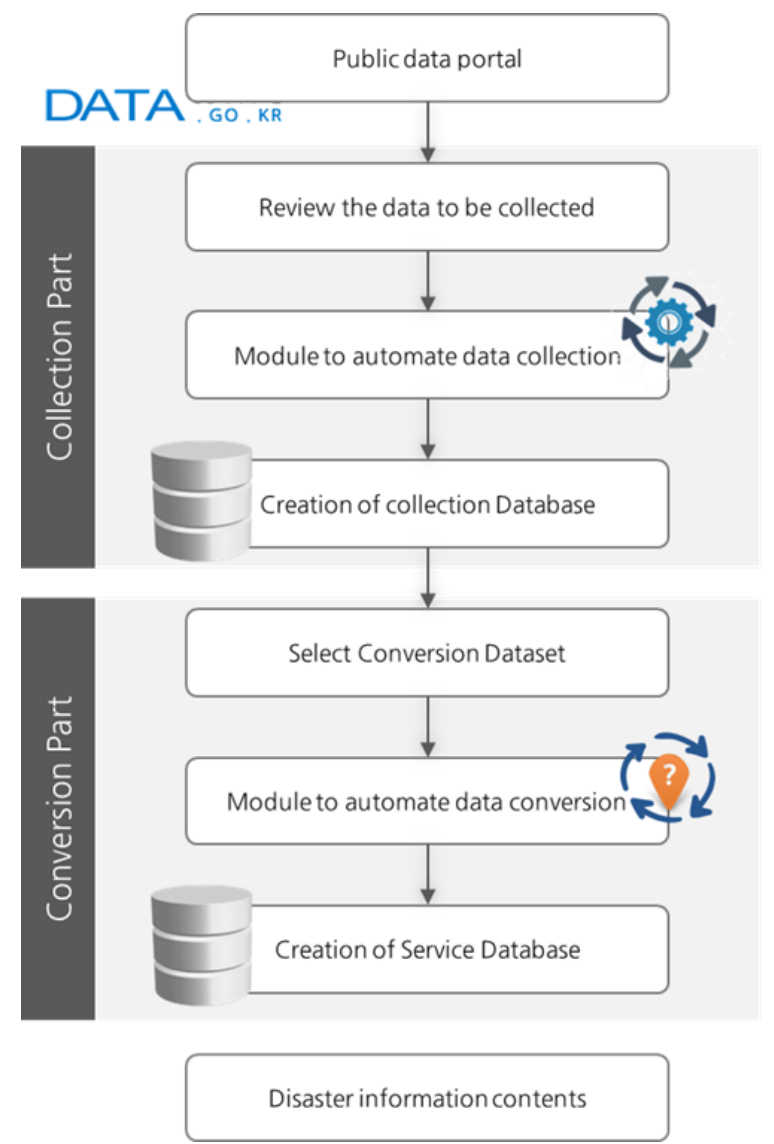

Figure 2. The Process for SFDIC construction

The process of collecting public data and converting it to SFDIC is shown in Figure 2. The SFDIC construction process can be roughly divided into two parts. The first part is for periodically collecting and storing the necessary data using the public data portal's API services. The data collection part uses the data open service of the public data portal to fetch the data and store it primarily in the database. Data collection targets data provided by open API method.

The second part is to select the necessary part in the primary stored database and convert it into the spatial information data. 
In this part, geocoding is performed using address or zip code information included in public data, and SFDIC is constructed. Geocoding is a technique for obtaining geographical coordinates using information that can be converted to a location. Since Korea implemented the road name address system in 2014 and used the parcel number system before, the address information of the public data is stored in either or both. Therefore, in order to convert public data into spatial data, a geocoding module to support both address and zip code is required. Using the coordinate information converted through this module, point type spatial data is generated. The SFDIC thus constructed can support various disaster management activities together with the SFDI map.

\subsection{Linkage system prototype}

For the verification of the process described in the previous section, it has developed a prototype ecosystem consisting of server module for data collection and conversion and web system for spatial information verification. The test data collected the data of the hospital, the public health center, and the general hospital in the health \& medical area, and converted it into the spatial information and constructed the SPDIC. The constructed data can be visualized as a map based on the web page as shown in Figure 3.

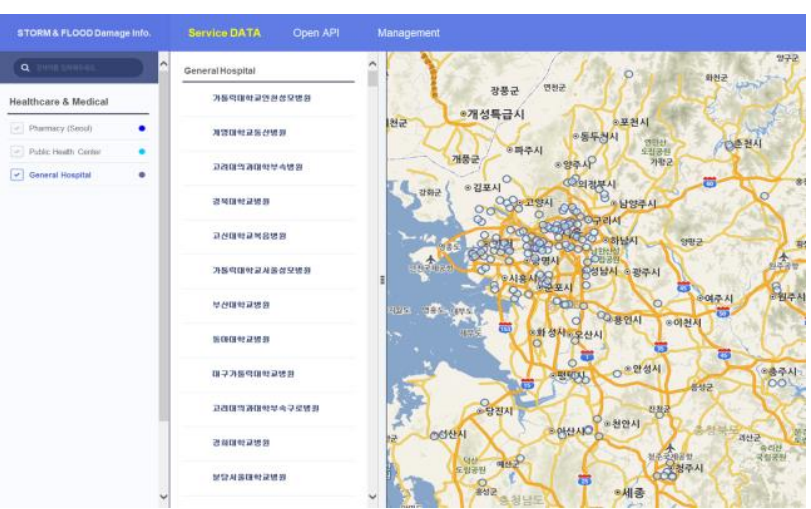

Figure 3. Figure placement and numbering

\section{CONCLUSION}

The SFDI map can be used not only for insurance information management purposes, but also for various policies related to prevention, preparedness, response and recovery of natural disaster. To do this, it is necessary to construct various related disaster information, which is related to various disaster information, in addition to SFDI map production. Open public data is an important source for efficient disaster information content construction. In this paper, a method for constructing SFDIC by collecting and converting this data has been proposed. Public data open policy is a global trend, and more data will be opened in various ways. Therefore, various researches are needed to utilize this data as disaster information.

\section{ACKNOWLEDGEMENTS}

This research was supported by a grant [MOIS-DP-2013-62] through the Disaster and Safety Management Institute funded by Ministry of the Interior and Safety of Korean government.

\section{REFERENCES}

Hwang, S., Sim, O., Kim, T., and Kim, W., 2015. Study of Effective Development Direction for the Storm and Flood Damage Insurance Management System through Analysis and Gathering of Requirements. Journal of Korea Society of Hazard Mitigation, 15(2), pp. 211-216.

Lee, J.S., Lee, I.S., 2015. A Study on Storm and Flood Damage Insurance Management Map Production. LXSIRI Report 18, Spatial Information Research Institute, Jeollabuk-do, Korea.

Lee, J.S., Lee, I.S., 2017. Automation Modelling of the Storm and Flood Hazard Risks for Insurance Premium Rate Map. Journal of the Korean Cadastre Information Association, 19(2), pp. 3-13.

Ministry of Public Safety and Security (MPSS), 2015 Statistical Yearbook of Natural Disaster, 2016, MPSS, Korea

Yeon, S., Kim, H., Lee, I., 2015. A Study on Geo-Data Appliance for Using Geospatial Information of Public Open Data. Journal of Cardastre \& Land InformatiX, 45(2), pp. 7185. 